\title{
Fatores associados ao desenvolvimento de audição e linguagem em crianças submetidas a implante coclear na região Norte
}

\author{
Factors associated with hearing and language development in children undergoing \\ cochlear implants in the Northern region \\ Factores asociados al desarrollo de la audición y el lenguaje en niños sometidos a \\ implantes cocleares en la región Norte
}

Ana Luiza Lopes de Freitas Freire ${ }^{1 *}$, Gisele Vieira Hennemann Koury ${ }^{1}$, Cíntia Tizue Yamaguchi ${ }^{1}$, Cecília Leite Gomes ${ }^{1}$, Karlla Lorenna dos Santos Anjos ${ }^{1}$, Diego Costa Farias ${ }^{1}$.

\begin{abstract}
RESUMO
Objetivo: Investigar os fatores relacionados ao desenvolvimento de audição e linguagem em crianças submetidas a cirurgia de Implante Coclear (IC) em um hospital universitário na região Norte do Brasil. Métodos: Estudo descritivo, transversal e retrospectivo de 48 pacientes submetidos ao IC, de 0 a 84 meses, entre 2010 e 2020. Foram descritos o estrato socioeconômico familiar, escolaridade dos pais, realização do teste da orelhinha, idade de entrada no serviço de IC, idade de implantação, acesso a fonoterapia pós IC e categorias de audição e linguagem alcançadas. Resultados: Pacientes submetidos ao teste da orelhinha $(40 \%)$ foram atendidos pela primeira vez no serviço de IC com idade menor e realizaram o procedimento em idade mais precoce. Crianças que tiveram acesso à fonoterapia pós IC apresentaram categorias de linguagem e audição superiores. A maior escolaridade dos pais se correlacionou com categorias de linguagem e audição melhores. Observou-se correlação significativa entre a idade de realização do IC, o estrato socioeconômico e a escolaridade dos pais. Conclusão: A identificação precoce da perda auditiva e a implantação no período crítico proporcionam melhores resultados auditivos e de linguagem.
\end{abstract}

Palavras-chave: Implante coclear, Audição, Reabilitação.

\begin{abstract}
Objective: To investigate factors related to hearing and language development in children undergoing Cochlear Implant $(\mathrm{Cl})$ surgery at a university hospital in Northern Brazil. Methods: Descriptive, cross-sectional and retrospective study of 48 patients undergoing $\mathrm{Cl}$, from 0 to 84 months, between 2010 and 2020. Family socioeconomic stratum, parents` education, performance on hearing screening, age at entry to the $\mathrm{Cl}$ service, age of implantation, access to speech therapy after $\mathrm{Cl}$ and hearing and language categories achieved. Results: Patients undergoing the hearing screening (40\%) were treated for the first time at the $\mathrm{Cl}$ service at a younger age and underwent the procedure at an earlier age. Children who had access to speech therapy after $\mathrm{Cl}$ had higher language and hearing categories. Higher parental education was correlated with better language and hearing categories. There was a significant correlation between age at $\mathrm{Cl}$ performance, socioeconomic status and parents' education. Conclusion: Early identification of hearing loss and implantation in the critical period provide better hearing and language results.
\end{abstract}

Keywords: Cochlear implantation, Hearing, Rehabilitation.

\section{RESUMEN}

Objetivo: Investigar factores relacionados con el desarrollo auditivo y del lenguaje en niños sometidos a cirugía de Implante Coclear (IC) en un hospital universitario del Norte de Brasil. Métodos: Estudio descriptivo, transversal y retrospectivo de 48 pacientes sometidos a IC, de 0 a 84 meses, entre 2010 y 2020 . Se describieron estratos socioeconómicos familiares, educación de los padres, realización de la prueba de oído, edad de ingreso al servicio de IC, edad de implantación, acceso a logopedia después de IC y categorías

\footnotetext{
${ }^{1}$ Hospital Universitário Bettina Ferro de Souza (HUBFS), Belém - PA. *E-mail: analuiza_freire@hotmail.com
} 
alcanzadas de audición y lenguaje. Resultados: Los pacientes sometidos a la prueba de oído (40\%) fueron tratados por primera vez en el servicio de IC a una edad más temprana y se sometieron al procedimiento a una edad más temprana. Los niños que tuvieron acceso a la terapia del habla después de la IC tenían categorías de lenguaje y audición más altas. La educación superior de los padres se correlacionó con mejores categorías de lenguaje y audición. Hubo una correlación significativa entre la edad en el desempeño de IC, el nivel socioeconómico y la educación de los padres. Conclusión: La identificación temprana de la pérdida auditiva y la implantación en el período crítico proporcionan mejores resultados de audición y lenguaje.

Palabras clave: Implantación coclear, Audición, Rehabilitación.

\section{INTRODUÇÃO}

O Implante Coclear (IC) foi considerado um dos mais importantes avanços da medicina moderna por possibilitar a habilitação e reabilitação da audição e a capacidade de comunicação de pessoas com surdez. Desde que as primeiras cirurgias de IC começaram a ser realizadas na população pediátrica, busca-se conhecer melhor quais seriam os fatores que podem influenciar (positiva e negativamente) os resultados obtidos com o IC, ou seja, o desenvolvimento das habilidades auditivas e de linguagem da criança e, consequentemente, o seu desenvolvimento biopsicossocial (SIAGH RFS, 2018).

Muitos fatores podem influenciar o desenvolvimento de audição e de linguagem oral, podendo ter um impacto isolado, mas também podendo interagir entre si, proporcionando diferentes graus de sucesso (BEVILACQUA MC, et al., 2005; GEERS AE, et al., 2007). Foi demonstrado que o tempo de privação auditiva é diretamente proporcional ao menor desempenho da percepção de fala. De modo que quanto mais precoce a cirurgia de IC foi realizada, melhores foram os resultados auditivos e de linguagem (TEAGLE HFB, et al., 2019).

Yoshinaga-itano C, et al. (2018) demonstraram a importância da triagem auditiva neonatal (TAN) precoce, de modo que o diagnóstico seja feito em tempo hábil e a implantação realizada o mais previamente possível. A TAN foi implementada pela Lei Federal no 12.303/2010 e torna obrigatória a realização do exame de Emissões Otoacústicas Evocadas em todos os hospitais e maternidades públicas (BRASIL, 2010).

O Joint Committee on Infant Hearing $(\mathrm{JCIH})$ preconizou que a TAN deve ser realizada até um mês de vida, o diagnóstico até os três meses de vida e o acesso ao tratamento com tecnologias de alta qualidade e complexidade, como Aparelhos de Amplificação Sonora Individuais (AASI) e IC, após o diagnóstico de perda auditiva permanente, até o sexto mês de vida (JOINT COMMITTEE ON INFANT HEARING, 2019).

Não foi descrito na literatura um consenso quanto ao período exato para realização de cirurgia de IC, no entanto, vários relatos têm apontado que sua realização em crianças menores de 1 ano pode ser segura e possibilitar resultados de desenvolvimento de fala semelhantes aos de crianças ouvintes. A tendência atual é a realização da cirurgia em crianças com menos de 2 anos, uma vez que quanto menor o período de privação auditiva, melhor será o desempenho dessa criança (TEAGLE HFB, et al., 2019; SHARMA SD, et al., 2020).

Siagh RFS (2018) relata que as crianças apresentam dificuldade em utilizar o Componente Externo (CE) de maneira sistemática, o que influencia negativamente no desenvolvimento auditivo e de linguagem, sendo o problema mais apontado o uso assistemático como opção da criança.

Lester EB, et al. (2011) relatam que as barreiras de saúde auditiva para crianças com deficiência auditiva incluem residência em zona rural, baixo nível de escolaridade dos pais e baixo nível socioeconômico. A falta de serviços de saúde auditiva, como observado nas áreas rurais, tem sido associada a atrasos no diagnóstico da perda auditiva (BUSH M, et al., 2015).

Foi demonstrado que a realização dos testes de triagem no período preconizado apresenta forte gradiente socioeconômico, sendo significativamente maior em crianças cujas famílias reportam maior renda domiciliar. Tal fato afeta diretamente a idade de realização do IC e, consequentemente, as categorias de audição e linguagem alcançadas (MALLMAN MB, et al., 2020). Foi demonstrado também que o nível de escolaridade materna tem um impacto direto sobre os resultados da linguagem, além de propiciar benefícios no diagnóstico e intervenção precoce do IC (YOSHINAGA-ITANO C, et al., 2018). 
Pacientes que possuem determinadas etiologias ou características, o grau de impacto do IC pode ser variado, como a meningite, infecções congênitas ou perinatais, prematuridade e intercorrências perinatais, a presença de atrasos motores associados, identificados na etapa pré-operatória, são descritos como fatores negativamente influentes no desempenho de audição e linguagem na etapa pós-operatória (BAMIOU DE, et al., 2001; BILLE J e OVESEN T, 2014).

Hassanzadeh S (2012) confirmou que as crianças surdas de segunda geração excedem as crianças surdas de pais ouvintes em termos de desempenho de IC. Além de comprovar que crianças surdas que se comunicam em linguagem de sinais desde muito cedo, antes do IC, melhoram sua habilidade de aprender a linguagem falada após o IC.

O objetivo do artigo foi investigar os fatores relacionados ao desenvolvimento de audição e linguagem em crianças submetidas a cirurgia de Implante Coclear (IC) em um hospital universitário na região Norte do Brasil permitiu vislumbrar o prognóstico da intervenção, além de adequar o aconselhamento à família, ajustando as suas expectativas quanto aos resultados a serem esperados, na busca de uma (re) habilitação de sucesso.

\section{MÉTODOS}

Trata-se de um estudo transversal, observacional e de caráter descritivo/analítico. A pesquisa foi realizada após a aprovação do Comitê de Ética em Pesquisa (CAAE: 47078721.2.0000.0018) em um hospital universitário sediado no município de Belém, no estado do Pará, sendo o único centro credenciado para realização de IC pelo Sistema Único de Saúde da região Norte do Brasil.

Foram inclusos na pesquisa crianças que foram submetidas a cirurgia de IC na faixa etária de 0 a 84 meses no período de 2010 a 2020, desde a implementação do serviço de IC na região norte, de modo a aumentar a amostra do estudo. Foram excluídos os pacientes em que a cirurgia de IC não foi realizada neste hospital, pacientes cujos responsáveis não permitiram o uso de seus dados ou cujos prontuários não contemplaram as informações requisitadas em protocolo de pesquisa.

Os dados foram coletados após a leitura e assinatura do Termo de Consentimento Livre e Esclarecido (TCLE) pelos pais ou responsáveis das crianças, a partir de prontuários e de um banco de dados próprio do serviço. As informações foram coletadas a partir de protocolo próprio elaborado pelos pesquisadores através da plataforma Google Forms.

Foram avaliadas as seguintes variáveis: sexo, cor/raça, idade, idade ao diagnóstico, idade na primeira consulta no serviço de IC e na implantação, fatores de risco pré-natais ou perinatais, comorbidades, dificuldade de acesso ao serviço de IC, resultado de teste da orelhinha, município e zona de residência, estrato socioeconômico (GRACIANO MIG e LEHFELD NAS, 2010). Além de grau de escolaridade do cuidador principal, categorias de audição e linguagem, horas de uso do componente externo do IC por dia, acesso a fonoterapia e aos retornos para programações pós implante, uso de outras formas de linguagem auxiliares e status auditivo dos pais.

A classificação de estrato socioeconômico foi realizada através de Instrumental de Avaliação Socioeconômica, que avalia: média salarial, número de membros da família, escolaridade, nível ocupacional, situação e condições habitacionais e outras questões subjetivas, finalizando com um sistema de pontuação que possibilita a definição da estratificação socioeconômica de cada família. Esta classificação dá-se em seis estratos, a saber: Baixo Inferior (BI), Baixo Superior (BS), Médio Inferior (MI), Médio (ME), Médio Superior (MS) e Alto (AL), sendo os mesmos determinados conforme a realidade apresentada pelo núcleo familiar (GRACIANO MIG e LEHFELD NAS, 2010).

Para caracterização do desenvolvimento das habilidades auditivas durante os anos de uso do IC, foram levantados dados da Categoria de Audição, proposta por Geers AE (1994), registrados nos prontuários pelos profissionais da Seção de IC na etapa pós-cirúrgica em cada retorno ao longo deste período.

As informações acerca da categoria de linguagem foram coletadas em prontuários a partir de avaliações realizadas pela equipe de IC. As categorias de linguagem foram classificadas conforme classificação proposta por Bevilacqua MC, et al. (2005). 
Posteriormente, os dados coletados foram organizados em um banco de dados. Para análise estatística, as variáveis categóricas foram descritas na forma de frequência absoluta e frequência relativa e o teste Quiquadrado foi usado para compará-las. Para análise de relação entre as variáveis contínuas, foi utilizado o teste de correlação de Spearman. Foram considerados valores estatisticamente significativos, testes com $p$ valor < 0,05. Foram utilizados o Software Microsoft Office Excel 16, Microsoft Word 365 e SigmaPlot 12.0.

\section{RESULTADOS E DISCUSSÃO}

No período compreendido entre junho e agosto de 2021, foram coletados dados de prontuários de 48 pacientes. A média de idade encontrada no momento da coleta de dados foi de 7,8 anos $\pm 2,8$ anos. Desses, $27(56,2 \%)$ pertencem ao sexo masculino e $21(43,7 \%)$ ao sexo feminino. A maioria foi classificada como parda $(87,5 \%)$, o que demonstra a representatividade da nossa população, corroborando dados da Pesquisa Nacional de Amostra de Domicílios (PNAD) de 2019, na qual a maioria da população se declarou parda (INSTITUTO BRASILEIRO DE GEOGRAFIA E ESTATÍSTICA, (IBGE), 2020).

A maioria dos pacientes era procedente do interior do Estado do Pará e possuía residência em área urbana (89,5\%). Noblitt B, et al. (2018), estudou 35 pacientes, sendo que os moradores de zona urbana (14 pacientes) possuíam acesso mais fácil e diverso a serviços de reabilitação da fala e consultas de retorno, demonstrando que ser morador de zona urbana configura-se como fator de melhor prognóstico para tratamento e seguimento.

Todos os pacientes eram filhos de pais ouvintes, não sendo possível a comparação das habilidades adquiridas entre crianças filhas de pais ouvintes e não ouvintes. Quanto aos estratos socioeconômicos, $54,1 \%$ pertenciam ao estrato $\mathrm{BI}$ e $41,6 \%$ ao BS.

A realização de pré-natal foi registrada para $91,6 \%$ da amostra avaliada e 16 pacientes apresentavam história de intercorrências gestacionais ou perinatais (Tabela 1). Este acompanhamento é fortemente difundido entre as políticas de saúde do Brasil, a fim de reduzir mortalidade materno-infantil e diversas comorbidades gestacionais, perinatais e infantis. Observou-se também que 16 dos participantes do estudo apresentaram intercorrências perinatais, sendo as mais encontradas: prematuridade, icterícia e internação em UTI neonatal (Tabela 1). Destas, apenas a permanência em UTI neonatal por mais de 5 dias e icterícia com necessidade de exsanguíneotransfusão configuram-se como fator de risco pelo Joint Committee on Infant Hearing (2019).

Tabela 1 - Características do Pré-Natal e Perinatal dos pacientes participantes do estudo.

\begin{tabular}{lcc}
\hline \multicolumn{1}{c}{ Características do Pré-Natal } & N (48) & $\%$ \\
\hline Realizou Pré-Natal & 44 & $91,6 \%$ \\
\hline Sim & 4 & $8,3 \%$ \\
\hline Aão & $\mathbf{N}(\mathbf{4 4 )}$ & $\%$ \\
\hline Sim & 16 & $36,3 \%$ \\
Não & 28 & $63,6 \%$ \\
\hline Intercorrências Gestacionais/Perinatais & $\mathbf{N ~ ( 1 6 )}$ & $\%$ \\
\hline Prematuridade & 6 & $37,5 \%$ \\
Icterícia & 5 & $31,2 \%$ \\
Internação em UTI & 3 & $18,7 \%$ \\
\hline
\end{tabular}

Fonte: Freire ALLF, et al., 2022.

A presença de doenças e comorbidades foram encontradas em 10 crianças, sendo as mais frequentes 0 transtorno do espectro autista (TEA) e a Síndrome de Waardenburg (Figura 1). Porém, a comorbidade mais associada à surdez é a infecção congênita por Citomegalovírus (CMV), a qual não foi detectada no presente estudo (SATTERFIELD-NASH A, et al., 2020). Tal fato pode ser explicado pois, conforme o Ministério da Saúde (2012), a rastreio de CMV não consta como exame obrigatório durante o pré-natal, o que gera grande subnotificação e falta de tratamento às gestantes e aos recém-nascidos portadores da doença (MINISTÉRIO DA SAÚDE, 2012). 
Figura 1 - Presença de Comorbidades entre os Pacientes entrevistados.

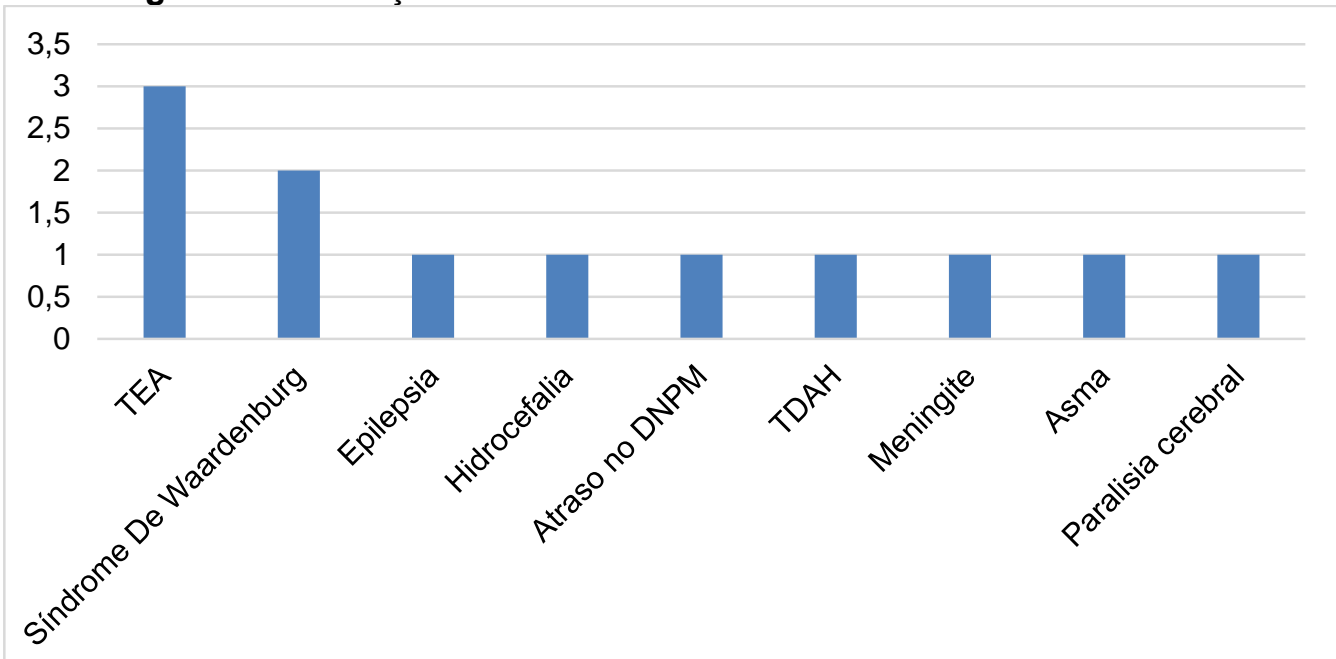

Legenda: TEA: Transtorno do espectro autista; DNPM: Desenvolvimento Neuropsicomotor; TDAH: Transtorno do Déficit de Atenção e Hiperatividade. Fonte: Freire ALLF, et al., 2022.

Foi encontrado relato de dificuldade no acesso ao serviço especializado em $89,5 \%$ dos prontuários dos pacientes. Após a realização do implante, $72,9 \%$ dos pacientes tiveram acesso aos retornos pós procedimento e $60,4 \%$ tiveram acesso à fonoterapia. Isso pode ser explicado pelo fato da maior parte dos participantes do estudo serem provenientes do interior do estado, localidades afastadas dos centros de referência localizados na capital. Além disso, esse dado serve de alerta ao número reduzido de serviços especializados de atenção terciária no âmbito da saúde auditiva na região Norte do país quando comparado a outras regiões, os quais não conseguem suprir de maneira efetiva a demanda populacional, diminuindo a adesão ao tratamento e aumentando os custos relacionados ao transporte de famílias a outras localidades para ter acesso ao serviço especializado (VIEIRA GI, et al., 2015). O uso de libras ou outra forma de linguagem foi relatado por apenas $4(8,3 \%)$ pacientes, o que pode ser explicado pelo fato de todos os pais serem ouvintes.

A média de idade em que os pacientes foram diagnosticados, realizaram sua primeira consulta no hospital universitário e, finalmente, foram submetidos à cirurgia de IC foram de $22 \pm 14$ meses, $30 \pm 18$ meses e $46 \pm$ 14 meses, respectivamente (Figura 2). Segundo a diretriz do Joint Committee on Infant Hearing (2019), o objetivo para as crianças é a identificação e a intervenção sendo iniciadas no máximo entre 3 a 6 meses de vida, demonstrando um atraso significativo tanto para o diagnóstico quanto para a intervenção cirúrgica no Norte do país. Desta forma, é importante mapear os pontos falhos no atual fluxo de atendimento a fim de estabelecer um fluxograma efetivo de trabalho que permita executar as ações de diagnóstico e intervenção na idade adequada.

Figura 2 - Média de idade dos pacientes avaliados em meses em diferentes momentos do acompanhamento clínico.

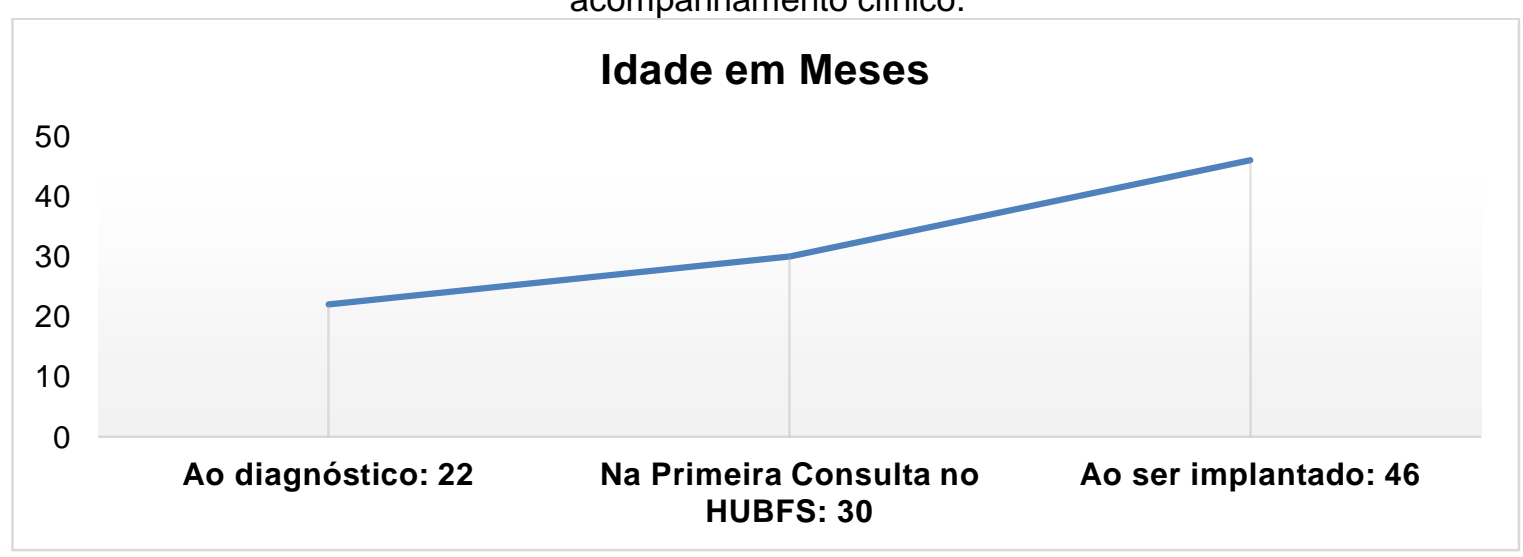

Fonte: Freire ALLF, et al., 2022. 
Quando foram avaliadas as categorias de audição e linguagem, foi possível perceber que a maior parcela da amostra era de pacientes das categorias 1 ou 2.

Ao comparar os pacientes que realizaram o teste da orelhinha $(39,5 \%)$ com aqueles que não realizaram $(60,4 \%)$, foi possível observar que os pacientes submetidos ao teste foram atendidos pela primeira vez no hospital universitário com idade significativamente menor. Observou-se que não houve significância estatística entre a realização do diagnóstico e a realização de IC em idade mais precoce entre os pacientes submetidos à triagem auditiva (Tabela 2).

Após o nascimento, são cruciais os testes de triagem neonatal, dos quais fazem parte o teste do pezinho, teste da orelhinha, teste do coraçãozinho e teste do olhinho. Mallman MB, et al., (2020) demonstraram que o gradiente socioeconômico influencia significativamente neste aspecto, haja vista que famílias com menor renda e menor escolaridade tinham menor acesso tanto ao pré-natal quanto à triagem auditiva neonatal. Tais dados corroboram as informações encontradas no presente estudo, haja vista que a maioria dos participantes pertence à estratos socioeconômicos majoritariamente baixos e apenas a minoria (40\%) teve acesso ao teste da orelhinha (Tabela 2).

A escolaridade dos pais se correlacionou com estrato socioeconômico $(r=0,443 ; p=0,002)$ e este, por sua vez, correlacionou-se com as categorias de linguagem $(r=0,337 ; p=0,039)$ e audição $(r=0,355 ; p=$ $0,026)$ dos pacientes. Também observamos correlação entre a idade de realização IC com o estrato socioeconômico $(r=-0,293 ; p=0,043)$ e com a escolaridade dos pais $(r=-0,334 ; p=0,020)$.

$\underline{\text { Tabela } 2 \text { - Comparação entre pacientes que realizaram teste da orelhinha com pacientes que não realizaram. }}$

\begin{tabular}{cccc}
\hline- & $\begin{array}{c}\text { Teste da Orelhinha } \\
\text { realizado }(\mathbf{N}=\mathbf{1 9})\end{array}$ & $\begin{array}{c}\text { Teste da Orelhinha não } \\
\text { realizado }(\mathbf{N}=\mathbf{2 9})\end{array}$ & $\mathbf{p}$ - valor \\
\hline $\begin{array}{c}\text { Idade ao diagnóstico } \\
\text { [mediana (1Q-3Q) em meses] }\end{array}$ & $16(7-30)$ & $24(12-30,5)$ & 0,472 \\
\hline $\begin{array}{c}\text { Primeira consulta no hospital } \\
\text { universitário }\end{array}$ & $16(5-45)$ & $33(26-40,5)$ & 0,035 \\
\hline $\begin{array}{c}\text { Realização do implante coclear } \\
\text { (média } \pm \text { desvio padrão em meses) }\end{array}$ & $41 \pm 16$ & $49 \pm 12$ & 0,055 \\
\hline
\end{tabular}

Fonte: Freire ALLF, et al., 2022.

Dentre os pacientes que realizaram o teste da orelhinha $(N=19)$, o resultado foi anormal em $12(63,1 \%)$. Este grupo específico se beneficiou altamente da realização do teste, uma vez que apresentou registro para idade significativamente menor em todos os momentos avaliado (idade do diagnóstico, primeira consulta e realização do implante), quando comparado aos grupos de pacientes com teste normal ou que não o realizaram (Tabela 3). Segundo Nikolopoulos TP (2015), antes da TAN, as crianças com problemas auditivos eram identificadas apenas aos 2 anos ou mais; neste estudo, as crianças que realizaram o teste foram diagnosticadas aos 11 meses, o que já corrobora os benefícios da TANU, sendo de extrema necessidade a conscientização de pediatras e profissionais de saúde que lidam com essas crianças (Tabela 3).

Mallman MB, et al. (2020), descreveu em seu estudo uma porcentagem de $65,8 \%$ de população que realizava o teste da orelhinha, enquanto $96,5 \%$ realizavam o teste do pezinho, diferença que pode ser explicada pelas populações analisadas, haja vista que a maioria da população em nosso projeto reside em áreas do interior do estado. Porém, em ambos os trabalhos é demonstrada a desigualdade no acesso aos testes da TAN. Tais desigualdades prejudicam significativamente os indivíduos portadores de deficiência auditiva, pois houve significância estatística ao avaliar a idade de diagnóstico, o acesso ao serviço especializado e a realização do implante coclear, sendo mais precoce em pacientes que realizaram a TAN (JOINT COMMITTEE ON INFANT HEARING, 2019).

Apesar de, neste estudo, não terem sido observadas correlações significativas entre as categorias de audição e linguagem e as idades de diagnóstico e intervenção, a literatura mostra que a idade de implantação é um preditor crucial dos benefícios do IC em crianças, visto que os efeitos da surdez no desenvolvimento do sistema auditivo e a neuroplasticidade remanescente mudam com a idade (SHARMA SD, et al., 2020). 
Tabela 3 - Comparação da idade ao diagnóstico, primeira consulta e realização do implante coclear, de acordo com o resultado do teste da orelhinha

\begin{tabular}{|c|c|c|c|c|}
\hline- & $\begin{array}{l}\text { Teste alterado } \\
\qquad(\mathrm{N}=12) \\
\end{array}$ & $\begin{array}{l}\text { Teste normal } \\
\qquad(\mathbf{N}=7)\end{array}$ & $\begin{array}{c}\text { Teste não } \\
\text { realizado } \\
(\mathrm{N}=29)\end{array}$ & $p$ - valor \\
\hline $\begin{array}{l}\text { Idade ao diagnóstico } 11 \pm 7 \\
\text { (média } \pm \text { DP em meses) }\end{array}$ & $11 \pm 7$ & $40 \pm 13$ & $23 \pm 11$ & $<0,001^{*}$ \\
\hline $\begin{array}{c}\text { Primeira consulta no hospital } \\
7,5(2,75-15,5) \\
\text { [mediana }(1 \mathrm{Q}-3 \mathrm{Q}) \text { em meses] }\end{array}$ & $7,5(2,75-15,5)$ & $47(27-59)$ & $33(26-40,5)$ & $<0,001^{\perp \tau}$ \\
\hline $\begin{array}{l}\text { Realização do implante coclear } \\
\text { (média } \pm \text { DP em meses) }\end{array}$ & $38 \pm 13$ & $54 \pm 14$ & $49 \pm 12$ & $<0,001^{\perp \tau}$ \\
\hline
\end{tabular}

Legenda: * Todos os grupos diferem entre si; + Grupo "Teste alterado" é diferente do grupo "Teste normal”; Grupo "Teste alterado" é diferente do grupo "Teste não realizado"

Fonte: Freire ALLF, et al., 2022.

Pacientes que tiveram acesso à fonoterapia pós IC $(N=29)$, apresentaram categorias de audição e linguagem significativamente melhores em comparação aos que não tiveram (Tabela 4). Isso é corroborado por Moretti C, et al. (2019), que demonstram que o benefício da inserção do IC é significativo no desenvolvimento da linguagem, haja vista que possibilita a percepção de sons de fala em frequência mais alta, possibilitando o reconhecimento e o desenvolvimento de linguagem oral mais rapidamente, sendo ideal a colocação da prótese ainda nos primeiros meses de vida. Porém, é notório que na população estudada, tanto o diagnóstico quanto o tratamento são realizados de forma tardia, o que gera prejuízos pessoais, sociais e econômicos aos pacientes.

Tabela 4 - Comparação das categorias de linguagem e audição de acordo com o acesso ou não à fonoterapia.

\begin{tabular}{lccc}
\hline & $\begin{array}{c}\text { Acesso à fonoterapia } \\
(\mathbf{N}=\mathbf{2 9})^{*}\end{array}$ & $\begin{array}{c}\text { Sem acesso à fonoterapia } \\
(\mathbf{N}=\mathbf{1 9})^{\boldsymbol{r}}\end{array}$ & $\mathbf{p}$ - valor \\
\hline $\begin{array}{l}\text { Categoria Linguagem } \\
\text { mediana (1Q-3Q) }\end{array}$ & $2(1-3)$ & $1(1-1,75)$ & $<0,05$ \\
\hline $\begin{array}{l}\text { Categoria Audição } \\
\text { mediana (1Q-3Q) }\end{array}$ & $2(1-4)$ & $1(1-2)$ & $<0,05$ \\
\hline
\end{tabular}

Legenda: * ${ }^{\prime}$ dados disponíveis para 26 pacientes; $\mathrm{r}=$ dados disponíveis para 12 pacientes.

Fonte: Freire ALLF, et al., 2022.

Quanto ao tempo de uso do dispositivo externo do implante coclear, a maioria (75\%) dos pacientes usava o dispositivo por menos de 8 horas/dia. Apesar de não ter apresentado significância estatística em nosso estudo, está intimamente relacionado aos progressos no desenvolvimento auditivo e de linguagem (ARCHBOLD SM, et al., 2009).

Os pacientes do interior do Estado $(\mathrm{N}=25)$ registraram porcentagem maior de acesso à fonoterapia $\mathrm{e}$ retorno pós IC quando comparados aos pacientes da Região Metropolitana de Belém $(N=19)$. Diante desse achado, faz-se necessário realizar algumas suposições, como a possibilidade desses pacientes apresentarem maior facilidade de marcação de consulta ou possuírem menos opções de terapia em seu município, de modo a terem que obrigatoriamente se deslocar até Belém. Deve-se atentar à possibilidade de menor oferta fonoterápica no município de Belém, de modo que os pacientes do interior do estado estejam realizando a fonoterapia em seu município de origem.

Diante dos resultados encontrados na pesquisa, faz-se necessário avaliar alternativas de agilizar o acesso destes pacientes dentro do fluxograma, diminuindo, sempre que possível, os empecilhos e burocracias que o sistema público de saúde, as condições socioeconômicas e o desconhecimento pela população dos prejuízos ocasionados pela perda auditiva precoce impõem. É de grande importância a conscientização da população, dos gestores da saúde e da equipe de profissionais envolvidos nesse processo, que devem atuar em conjunto, de maneira interdisciplinar, agilizando todas as etapas de assistência à saúde desse paciente, sempre que possível. 


\section{CONCLUSÃO}

A maioria das crianças submetidas ao IC neste serviço eram do sexo masculino, de cor parda e procedentes do interior do Pará. As intercorrências gestacionais e perinatais mais prevalentes foram prematuridade, icterícia e internação em UTI. As comorbidades mais prevalentes foram o TEA e a síndrome de Waardenburg. A média de idade de realização do IC foi de 46 meses. Os fatores que influenciaram para o diagnóstico, realização das consultas e seguimento foram o acesso à TAN, a escolaridade e o estrato socioeconômico dos pais. A maioria usava O CE do IC menos de 8 horas por dia e apresenta-se com categorias de linguagem e audição entre 1 e 2. $O$ trabalho possibilitou o conhecimento da realidade das crianças implantadas na região norte e o vislumbre de intervenções específicas para essa população.

\section{REFERÊNCIAS}

1. ARCHBOLD SM, et al. Long-term use of cochlear implant systems in paediatric recipients and factors contributing to non-use. Cochlear Implants International, 2009; 10(1): 25-40.

2. BAMIOU DE, et al. Eighth nerve aplasia and hypoplasia in cochlear implant candidates: the clinical perspective. Otology \& Neurotology, 2001; 22(4): 492-496.

3. BEVILACQUA MC, et al. Considerações sobre o implante coclear em crianças. In: Bevilacqua MC, Moret ALM. Deficiência auditiva: conversando com familiares e profissionais da saúde. $1^{\underline{a}}$ ed. São José dos Campos: Editora Pulso, 2005; p.123-138.

4. BILLE J, OVESEN T. Cochlear implant after bacterial meningitis. Pediatrics International, 2014; 56(3): $400-405$.

5. BRASIL. Lei 12.303 , de 02 de agosto de 2010. Dispõe sobre a obrigatoriedade de realização do exame denominado Emissões Otoacústicas Evocadas. Brasília, DF: Presidência da República, 2010. Disponível em: http://www.planalto.gov.br/ccivil_03/_ato2007-2010/2010/lei/l12303.htm. Acessado em: 30 de janeiro de 2022.

6. BUSH M, et al. Rural barriers to early diagnosis and treatment of infant hearing loss in Appalachia. Otology \& Neurotology, 2015; 36(1): 93-98.

7. GEERS AE, et al. Estimating the Influence of cochlear implantation on language development in children. Audiological Medicine, 2007; 5(4): 262-273.

8. GEERS AE. Techniques for assessing auditory speech perception and lipreading enhancement in young deaf children. Volta Review. 1994; 96(5): 85-96.

9. GRACIANO MIG, LEHFELD NAS. Estudo socioeconômico: indicadores e metodologia numa abordagem contemporânea. Serviço Social e Saúde, 2010; 9(1): 157-186.

10. HASSANZADEH S. Outcomes of cochlear implantation in deaf children of deaf parents: comparative study. The Journal of Laryngology \& Otology, 2012; 126(10): 989-994.

11. INSTITUTO BRASILEIRO DE GEOGRAFIA E ESTATÍSTICA. Pesquisa Nacional por Amostra de Domicílios Contínua (PNAD) COVID-19. Microdados. Rio de Janeiro: IBGE; 2020. Disponível em: https://www.ibge.gov.br/estatisticas/sociais/trabalho/27946-divulgacao-semanal-pnadcovid1.html. Acessado em: 30 de janeiro de 2022.

12. JOINT COMMITTEE ON INFANT HEARING. Year 2019 Position Statement: Principles and Guidelines for Early Hearing Detection and Intervention Programs. Journal of Early Hearing Detection and Intervention, 2019; 4(2): 1-44.

13. LESTER EB, et al. Barriers to the early cochlear implantation of deaf children. Otology \& Neurotology, 2011; 32(3): 406-412.

14. MALLMANN MB, et al. Neonatal screening tests in Brazil: prevalence rates and regional and socioeconomic inequalities. Jornal de Pediatria, 2020; 96(4): 487-494.

15. MINISTÉRIO DA SAÚDE. Secretaria de Atenção à Saúde. Departamento de Ações Programáticas Estratégicas. Diretrizes de Atenção da Triagem Auditiva Neonatal. Brasília-DF: Brasil, 2012. Disponível em: https://bvsms.saude.gov.br/bvs/publicacoes/diretrizes_atencao_triagem_auditiva_neonatal.pdf. Acessado em: 30 de janeiro de 2022.

16. MORETTI C, et al. Brazilian Scale of Hearing and Language Development: Normality Curve for Infants and Children from 0 to 24 Months Old with Normal Hearing. International Archives of Otorhinolaryngology, 2019; 23(2): $131-136$.

17. NIKOLOPOULOS TP. Neonatal hearing screening: what we have achieved and what needs to be improved. International Journal of Pediatric Otorhinolaryngology, 2015; 79(5): 635-637.

18. NOBLITT B, et al. Barriers to Rehabilitation Care in Pediatric Cochlear Implant Recipients. Otology \& Neurotology, 2018; 39(5): e307-e313.

19. SATTERFIELD-NASH A, et al. Etiology of Prelingual Hearing Loss in the Universal Newborn Hearing Screening Era: A Scoping Review. Otolaryngology-Head and Neck Surgery, 2020; 163(4): 662-670.

20. SHARMA SD, et al. Hearing and speech benefits of cochlear implantation in children: A review of the literature. International Journal of Pediatric Otorhinolaryngology, 2020; 133:109984.

21. SIAGH RFS. Crianças usuárias de implante coclear com atraso do desenvolvimento da percepção auditiva da fala: análise dos fatores que influenciam o desempenho. Tese (Doutorado em Fonoaudiologia) - Faculdade de Odontologia de Bauru, Universidade de São Paulo, Bauru, 2018.

22. TEAGLE HFB, et al. Pediatric cochlear implantation: A quarter century in review. Cochlear Implants International, 2019; 20(6): 288-298.

23. VIEIRA GI, et al. Saúde Auditiva no Brasil: análise quantitativa do período de vigência da Política Nacional de Atenção à Saúde Auditiva. Distúrbios da Comunicação, 2015; 27: 725.

24. YOSHINAGA-ITANO C, et al. Language Outcomes Improved Through Early Hearing Detection and Earlier Cochlear Implantation. Otology \& Neurotology, 2018; 39(10): 1256-1263. 